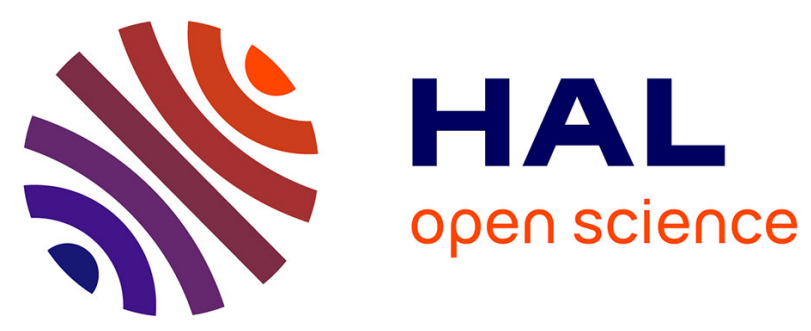

\title{
AR Feels "Softer" than VR: Haptic Perception of Stiffness in Augmented versus Virtual Reality
}

Yoren Gaffary, Benoît Le Gouis, Maud Marchal, Ferran Argelaguet Sanz, Anatole Lécuyer, Bruno Arnaldi

\section{- To cite this version:}

Yoren Gaffary, Benoît Le Gouis, Maud Marchal, Ferran Argelaguet Sanz, Anatole Lécuyer, et al.. AR Feels "Softer" than VR: Haptic Perception of Stiffness in Augmented versus Virtual Reality. IEEE Transactions on Visualization and Computer Graphics, 2017, 23 (11), pp.2372 - 2377. 10.1109/TVCG.2017.2735078 . hal-01625290

\section{HAL Id: hal-01625290 https://hal.inria.fr/hal-01625290}

Submitted on 27 Oct 2017

HAL is a multi-disciplinary open access archive for the deposit and dissemination of scientific research documents, whether they are published or not. The documents may come from teaching and research institutions in France or abroad, or from public or private research centers.
L'archive ouverte pluridisciplinaire HAL, est destinée au dépôt et à la diffusion de documents scientifiques de niveau recherche, publiés ou non, émanant des établissements d'enseignement et de recherche français ou étrangers, des laboratoires publics ou privés. 


\section{AR Feels "Softer" than VR: Haptic Perception of Stiffness in Augmented versus Virtual Reality}

\author{
Yoren Gaffary*i \\ Inria/IRISA \\ Ferran Argelaguet ${ }^{\dagger}$ \\ Inria/IRISA
}

\author{
Benoît Le Gouis*† \\ INSA Rennes/IRISA \\ Bruno Arnaldi ${ }^{\dagger}$ \\ INSA Rennes/IRISA
}

\author{
Maud Marchal ${ }^{\dagger}$ \\ INSA Rennes/IRISA \\ Anatole Lécuyer ${ }^{\dagger}$ \\ Inria/IRISA
}

\begin{abstract}
Does it feel the same when you touch an object in Augmented Reality (AR) or in Virtual Reality (VR)? In this paper we study and compare the haptic perception of stiffness of a virtual object in two situations: (1) a purely virtual environment versus (2) a real and augmented environment. We have designed an experimental setup based on a Microsoft HoloLens and a haptic force-feedback device, enabling to press a virtual piston, and compare its stiffness successively in either Augmented Reality (the virtual piston is surrounded by several real objects all located inside a cardboard box) or in Virtual Reality (the same virtual piston is displayed in a fully virtual scene composed of the same other objects). We have conducted a psychophysical experiment with 12 participants. Our results show a surprising bias in perception between the two conditions. The virtual piston is on average perceived stiffer in the VR condition compared to the AR condition. For instance, when the piston had the same stiffness in $\mathrm{AR}$ and VR, participants would select the VR piston as the stiffer one in $60 \%$ of cases. This suggests a psychological effect as if objects in AR would feel "softer" than in pure VR. Taken together, our results open new perspectives on perception in AR versus VR, and pave the way to future studies aiming at characterizing potential perceptual biases.
\end{abstract}

Keywords: Augmented Reality, Virtual Reality, Haptic, Perception, Stiffness, Psychophysical Study.

Index Terms: H.5.1 [Information Systems]: Information Interfaces and Presentation-Artificial, augmented, and virtual realities; H.5.2 [Information Systems]: Information Interfaces and PresentationHaptic I/O

\section{INTRODUCTION}

Virtual Reality and Augmented Reality are gaining more and more interest in the general audience as well as in the research community. $\mathrm{AR}$ and VR rely on similar technologies, but they provide a different kind of visual feedback. A main difference is the presence, or not, of real objects in the field of view of the user. However, as for today, it remains unclear if this difference can influence the perception of the user. In other words, how different is perception in AR from perception in VR?

Visual perception in AR has been rather widely studied, taking into account several parameters such as the environment, the augmentation, the display device, or even the user [12]. Some biases in visual perception well-documented in VR, such as distance underestimation, have also been observed in AR [8], with a lesser magnitude. However, though there exist previous studies on visual perception in

*Both authors contributed equally to this work.

†e-mail:firstname.lastname@inria.fr
AR and its difference with VR, there are actually very few studies on other sensory modalities, and in particular on the haptic sense.

Is haptic perception in AR different from haptic perception in VR? The presence of real objects in AR might indeed influence the way we interact with virtual objects and, eventually, the way we perceive them. In the end, the question we raise here is: does it feel the same when you touch an object in Augmented Reality or when you touch it in Virtual Reality?

In this paper, we study how haptic perception of stiffness of a virtual object is influenced by displaying the scene in AR versus in VR. We conducted an experiment based on a Microsoft HoloLens in which participants could interact with an object (a virtual piston) inside a real scene and inside a virtual reproduction of the same scene. The participants were able to press on the virtual piston and perceive its stiffness using a force-feedback haptic device. They could successively compare the stiffness of the virtual piston in $\mathrm{AR}$ and in VR, with various levels of stiffness difference. The results show that, on average, participants perceived the virtual piston as "stiffer" in the virtual environment than in the augmented environment.

In the remainder of this paper, we first present related work on perception in VR and AR in Section 2. Then, in Section 3, we describe the protocol and apparatus of our experimental study. The results obtained are presented in Section 4 followed by a discussion in Section 5. The paper ends with a general conclusion in Section 6

\section{Related Work}

The study of human perception in virtual and/or augmented reality and its comparison with perception in reality has been an active field of research since many years [18] [3] [12]. In particular, a difference in distance estimation has been early reported between real and virtual environments [18]. Objects in VR look closer than they actually are. Although the visual feedback provided in AR differs greatly from VR, the same perceptual bias concerning depth or distance estimation has also been observed in AR [8]. Then, Knorlein et al. [11] observed that a delay in force-feedback in AR could give the impression that a virtual object was softer. A similar effect had also been found in a VR context [16].

Visual feedback in VR and AR is known to influence haptic perception [13]. The phenomenon of "visual dominance" was notably observed when estimating the stiffness of virtual objects. In a pioneer study, Srinivasan et al. [19] showed that the distorted visual display of a spring elongation could strongly bias the stiffness perceived when manipulating a haptic force-feedback device. Later on, Lécuyer et al. based their "pseudo-haptic feedback" approach on this notion of visual dominance [13] [15] [14]. They notably showed how playing with visual feedback enables to simulate a wide range of stiffness sensations when using a passive elastic device [15]. The researchers noticed a perceptual offset between the perception of a real spring and the perception of such a pseudo-haptic spring simulated with visual feedback. The pseudo-haptic spring was globally underestimated compared to the real spring. Using a psychophysical method, they found that the perceptual offset (or Point of Subjective 
Equality) was on average equal to $9 \%$. Interestingly enough, other perceptual biases have also been observed regarding haptic perception of stiffness such as a depth or perspective effect [21]. This effect implies that objects located at a farther distance are perceived as stiffer [21].

Several other previous works have focused on how the perceived haptic properties of a real object could be changed by a visual superimposition of information on this object. Hirano et al. [6] have notably superimposed textures associated with different levels of hardness on a real object, successfully influencing the perception of hardness of this object. Similar methods have been proposed to influence a "softness" perception [17] or the perceived weight of an object ( [5]. In a purely AR context, Jeon and Choi [7] have also shown how adding a force-feedback during interaction with a real object could modulate the stiffness perceived by the user.

Haptic stiffness perception has been widely investigated in VR environments. These works use psychophysical methods to study perception, and compute two perceptual variables, the Just Noticeable Difference (JND) and the Point of Subjective Equality (PSE). The JND is the point at which there is no perceptual difference between two stimuli, ie every stimulus with a relative difference to a reference stimulus inferior to the JND is perceived as equal to the reference one. The PSE is used to compare two stimuli of different natures, and corresponds to the point where they are perceived as exactly equal. The stimuli that are perceived as equal are thus in a range of values centered at the PSE value, with a span of 2 times the JND. The JND for stiffness perception has been widely investigated, with variations in the studied limbs, stiffness ranges, and stimulus nature. For instance, Jones et al. [9] have studied stiffness perception during the interaction with the arm and important stiffnesses, up to $6.26 \mathrm{~N} . \mathrm{mm}^{-1}$. Cholewiak et al. [1] have studied perception on the wrist, with stiffnesses up to $3 \mathrm{N.mm}$, and Gugari et al. 4 studied perception of the finger, with stiffnesses up to $0.34 \mathrm{~N} . \mathrm{mm}^{-1}$ Overall the JND range is found to be between $15 \%$ and $22 \%$ [10]. Other works have focused on adding modalities to the haptic stiffness perception. For instance, vibrations were found to increase softness sensation [20].

However, to the best of authors' knowledge there is no previous work which specifically compared haptic perception in Augmented Reality versus Virtual Reality.

\section{User study: Haptic Perception of Stiffness in VR Versus AR}

This experiment aims at studying the potential influence of visual display, i.e. using Virtual Reality versus Augmented Reality, on the haptic perception of a virtual object (a piston). More specifically, we studied the influence of the nature of the visual surrounding of the piston (real or virtual) on its perceived stiffness. Participants had then to compare the stiffness of two pistons displayed sequentially. One of the piston was displayed in AR and the other one in VR, in a counterbalanced order.

The reader is encouraged to look at the accompanying video for a comprehensive description of the experimental apparatus and procedure.

\subsection{Participants}

12 participants (11 males, 1 female) took part in the experiment. They were aged from 20 to $29($ mean $=23.7, \mathrm{SD}=3.2)$. All of them were right-handed.

\subsection{Experimental apparatus}

The display of the virtual elements in AR and VR environments was achieved using a Microsoft HoloLens visual display a see-through

1 WWW . microsoft . com/microsoft-HoloLens

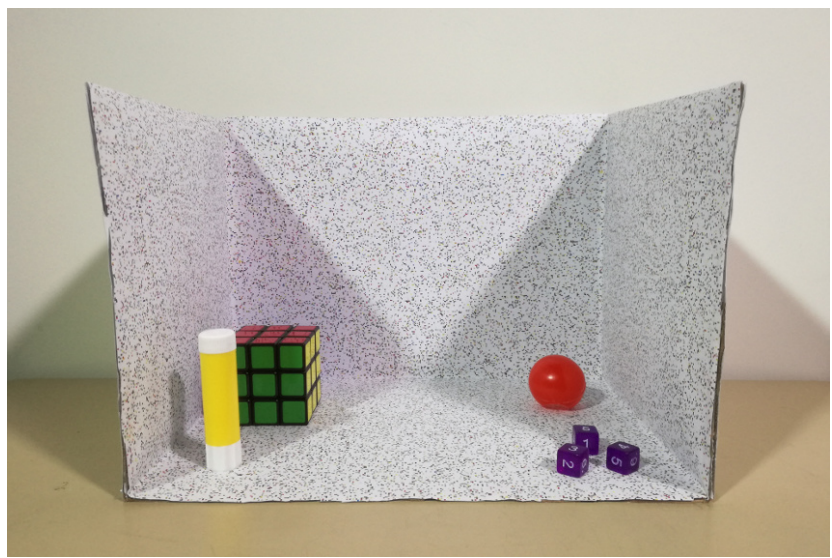

Figure 1: Close-up of the scene. A cardboard box with a colored texture (random colored dots) contains several casual objects: a yellow glue stick, a Rubik's cube, a red clown nose, and three violet dice.

HMD that can superimpose images on a portion of the field of view, with built-in tracking possibilities.

The experimental setup is then based on a visual scene composed of a cardboard box containing several objects with simple shapes: a glue stick, a Rubik's cube, a red clown nose and three violet dice (see Figure 1). The inner faces of the box were covered with printed sheets of paper displaying colored random dots. The lighting was carefully provided by two LED projectors as to: (1) fully illuminate the scene, and (2) provide sufficient light levels for the real scene to be brightly lit, but not too strong for the HoloLens to be able to occlude efficiently the real environment in VR.

Participants were comfortably seated 2-meters in front of the cardboard box, at a distance that allowed to see all the scene at once with the HoloLens, yet with sufficient details (see Figure 3). In order to provide the participants with the same field of view in both AR and VR environments using the HoloLens, the peripheral field of view was hidden using a mask made of a piece of tissue with two rectangular holes for the eyes and attached to the HoloLens. Thus, the remaining field of view matches the field of view of the HoloLens and could be fully superimposed with the virtual scene.

The scene was entirely reproduced in a faith-full manner in VR, including: the cardboard box, the objects, the front wall, the table and the lighting conditions. In the VR environment, due to the good occlusion capacities of the HoloLens and the careful handling of the lighting, the real scene was almost invisible. A virtual piston was then superimposed on the real scene in AR, or integrated to the virtual environment, as depicted in Figure 2

Participants could interact using their dominant hand with the virtual piston using a haptic force-feedback device (Falcon, Novint company). They manipulated a 3D cursor (represented as a 3D blue sphere) along 3 degrees of freedom, with a 1:1 mapping between the motion of the haptic device extremity and the motion of the $3 \mathrm{D}$ cursor. Once the 3D cursor was in contact with the virtual piston the participants could exert pressure on it. The stiffness of the piston was then rendered using the force-feedback and simulating a pure spring along the vertical axis. The Falcon device was positioned sideways in order to ensure higher forces and a more homogeneous haptic manipulation workspace. The haptic rendering was handled by a remote computer using CHAI3D software API ${ }^{2}$ and the position of the haptic device was streamed to the HoloLens using WiFi. Participants also used a numerical pad attached using a band to their left leg with two keys labelled " 1 " and " 2 ", in order to answer the 


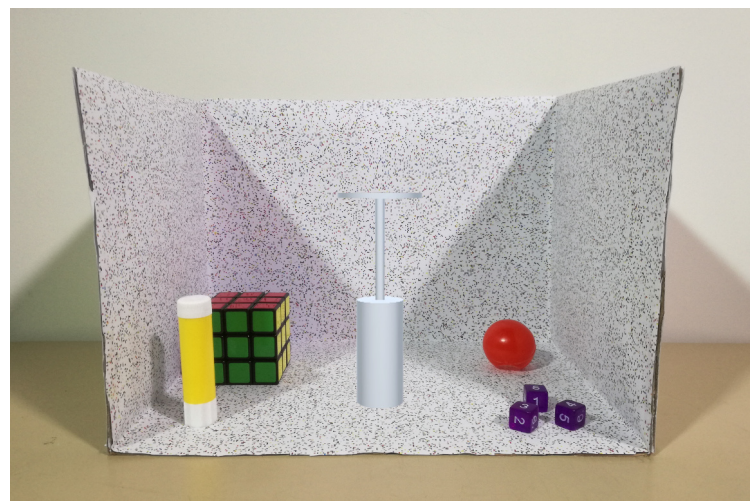

(a) Virtual piston in AR.

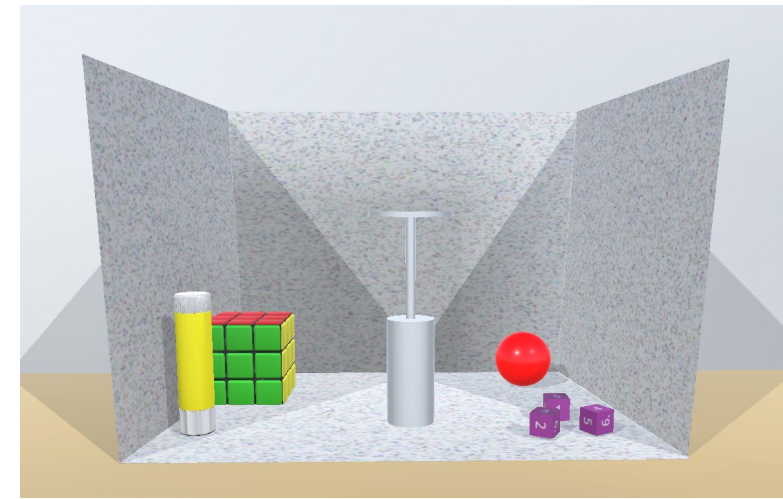

(b) Virtual piston in VR.

Figure 2: Experimental conditions. (a) AR environment with a virtual piston superimposed inside the real cardboard box. (b) VR environment with the same virtual piston located inside the virtual scene.

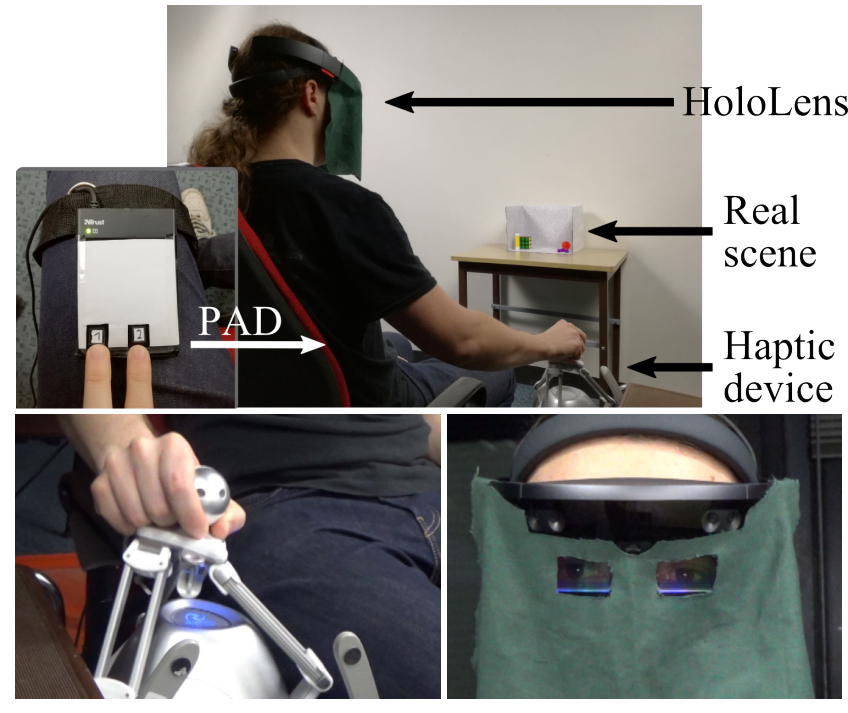

Figure 3: Experimental setup. The participant is seated in a comfortable chair wearing a HoloLens device and uses a pad to answer which of two virtual pistons was the stiffest (top). He interacts with a virtual piston using a Novint Falcon haptic device located at his side (bottom-left). A mask (bottom-right) with two holes for the eyes and made of tissue is fixed on the HoloLens so to hide the peripheral field of view which cannot be augmented by the HoloLens.

questions in a comfortable manner with their left hand.

The choice of the non co-located interaction was motivated by two major constraints, first, the field of view of the HoloLens and second, the integration of the haptic device and the hand of the participants in the VR condition. The proposed setup enables the entire scene to be visible from the HoloLens and avoids the integration of the haptic device and the participant's hand. Although having a non colocated interaction might have an influence on the haptic perception [2], this effect would equally affect the perception in both AR and VR conditions and should thus not drastically alter the experiment results.

\subsection{Conditions and Plan}

There were two environment conditions related to the visual display. The AR condition corresponds to the use of an augmented reality display mode, whereas the VR condition corresponds to the use of a virtual reality display mode. In addition, two other conditions are considered:

- $\mathbf{C 1}$ is the visual condition of the reference piston. AR reference means that the reference piston is displayed in AR, and VR reference means that the reference piston is displayed in VR. The value of the stiffness of the reference condition was set in both cases to $0.11 \mathrm{~N} . \mathrm{mm}^{-1}$ after preliminary testings.

- $\mathbf{C 2}$ is the stiffness value of the comparison piston. Five possible values were chosen after preliminary testings, corresponding to the following five differences: $-16 \%,-8 \%, 0 \%,+8 \%$ and $+16 \%$ compared to the reference stiffness.

The order of presentation of the two pistons and their display environment were counterbalanced to avoid any order effect [22]: every couple of pistons was presented in all orders (AR first/VR first, reference first/comparison first).

Thus, participants were presented with 100 trials, divided in 5 blocks of 20 trials in a different randomized order for each block. Each block of 20 trials presented a set of couples of pistons made of: 2 stiffness reference $(\mathbf{C 1}) \times 5$ stiffness values $(\mathbf{C 2}) \times 2$ presentation orders (AR then VR, or VR then AR)).

\subsection{Procedure}

Participants started by filling out a short form. After verbal explanations, they performed 5 training trials during which they could get used to the experiment procedure. Then, the participants were presented with the set of 100 trials. The procedure for each trial is as follows (see also Figure 4).

A real scene (AR condition) or virtual scene (VR condition) was displayed (see Figure 2), all including a virtual piston and a 3D cursor (blue sphere). A red cylinder located over the piston represented the starting position volume, as depicted in Figure $4 \mathrm{a}$ The participants had to reach and remain in the starting position volume for $1 \mathrm{~s}$ before being able to interact with the piston. After that delay, the cylinder turned green (Figure 4b), and the participant could interact with the piston for $3 \mathrm{~s}$, as seen in Figure $4 \mathrm{c}$ At the end of the exploration time, a panel with a stop message was displayed in front of the scene, and the red cylinder reappeared (Figure 4d). When the participants reached again the red cylinder, the condition (AR or VR) changed, as well as the stiffness of the second piston. The participant still needed to stay inside the red cylinder (starting position volume) for $1 \mathrm{~s}$ before being able to interact again with 


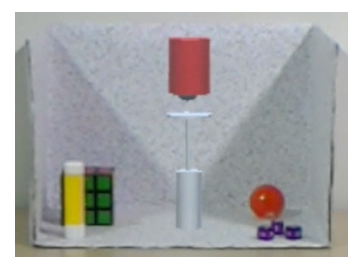

(a)

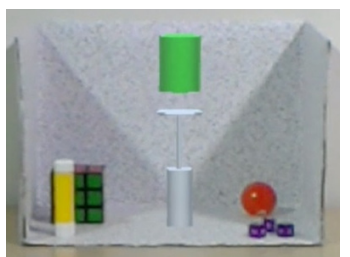

(b)

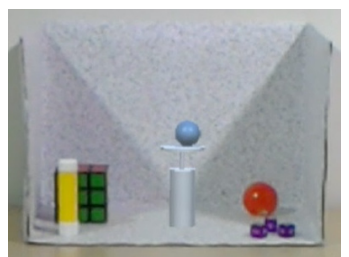

(c)

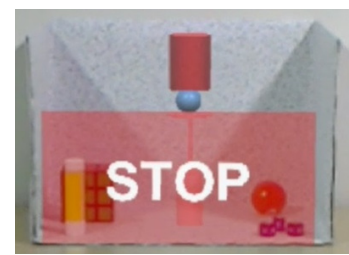

(d)

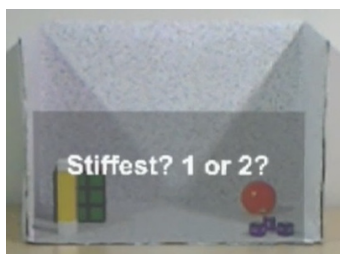

(e)

Figure 4: Experimental procedure (displayed here in the AR condition). (a) A red cylinder displays the starting position to reach with the manipulated cursor (blue sphere). (b) The cylinder turns to green to inform the participant that he/she can start evaluating the stiffness of the first piston. (c) The participant can press on the piston using the manipulated cursor. (d) A stop sign and panel indicates that the evaluation time is over. The same sequence (a-b-c) is then proposed in the second condition (VR here). Then, (e) The participant must answer, ie report which piston is the stiffer. Pictures were captured using the HoloLens camera.

the second piston. After $3 \mathrm{~s}$ of interaction with the second spring, the stop panel reappeared, and after reaching the starting position volume for the third time, the participant was presented with the response panel asking which was the stiffest pistons (1 or 2), as shown in Figure $4 \mathrm{e}$ The participants could enter their answer using the pad attached to their left leg, as displayed in Figure 3 Once the answer of the participant was recorded the next trial started. After each block of 20 trials, a break was proposed to the participant.

\subsection{Collected data}

For each couple of pistons, we collected 5 objective measures:

- Om1: Participant's answer is the piston $\left(1^{\text {st }}\right.$ or $\left.2^{\text {nd }}\right)$ which was reported by the participant as the stiffer one.

- Om2: Response time corresponds to the elapsed time between the end of the evaluation of the second piston and the moment the participant entered his/her answer.

- Om3: Displacement quantity corresponds to the sum of every vertical displacement (absolute value, in meters) of the haptic device when in contact with the piston during the interaction. This measure was recorded separately for the two presented pistons.

- Om4: Force corresponds to the average force (in N) the participants received from the device over the interaction time. This measure was recorded separately for the two presented pistons.

Participants also completed a subjective questionnaire at the end of the experiment. Each question of this questionnaire was answered using a 7-item Likert scale:

- Sm1: "The piston seemed real in augmented reality."

- Sm2: "The piston seemed real in virtual reality."

- Sm3: "I did not see the real environment when the scene was entirely virtual". This question was asked to evaluate the quality of the occlusion of the real scene in the VR condition.

- Sm4: "Except for their real/virtual aspect, I did not notice any difference between the augmented and the virtual scenes". This question was asked to evaluate the correctness of the reproduction of the virtual scene compared to the real scene.

- Sm5: “After the experiment, I felt visual fatigue."

- Sm6: "After the experiment, I felt haptic fatigue."

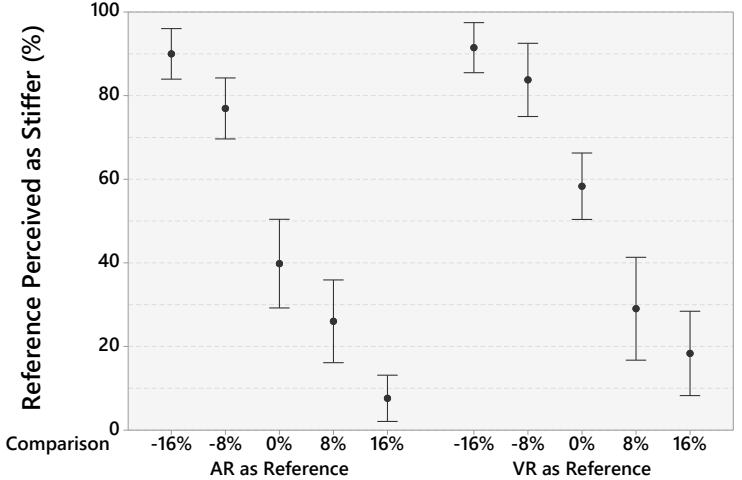

Figure 5: Percentage of times that the reference object is chosen (Confidence intervals at 95\%) when asking "which piston was stiffer?" for the five comparison conditions and the two environment references.

We also asked an open question to the participants $\mathbf{S m 7}$ "Do you think that the real environment influenced your haptic perception of the virtual piston? If so, how?". This question was asked to get a subjective feedback concerning the possible influence of the type of environment on the stiffness perception.

\section{Results}

\subsection{Recognition Accuracy}

In order to analyze the participants' answers (Om1) we pooled the data for all repetitions (no ordering effects were found). For each combination of factors, we computed the percentage of the answers in which the reference piston was perceived to be stiffer than the comparison piston, then we performed a two-way ANOVA analysis considering the nature of the reference environment (C1) and the stiffness of the comparison object $(\mathbf{C 2})$ (see Figure 5). The ANOVA showed a main effect for $\mathbf{C 1}\left(F_{1,11}=15.72 ; p<0.01\right.$; $\left.\eta_{p}^{2}=0.59\right)$. Post-hoc tests showed that when the environment was virtual, the reference object was significantly considered stiffer $M=0.51 ; S D=0.33$ than when the environment was real $M=0.44$; $S D=0.32$. This rejects the null hypothesis, which is "there is no difference between the two conditions".

In addition, we also observed a main effect for $\mathbf{C 2}$ on the stiffness of the comparison object $\left(F_{4,44}=100.48 ; p<0.001 ; \eta_{p}^{2}=0.90\right)$. As expected, as the stiffness of the comparison object increases, the number of trials that the reference piston is considered to be stiffer decreases (see Figure 5). The ANOVA did not show any interaction effect $\left(F_{4,44}=1.95 ; p=0.119 ; \eta_{p}^{2}=0.15\right)$. 


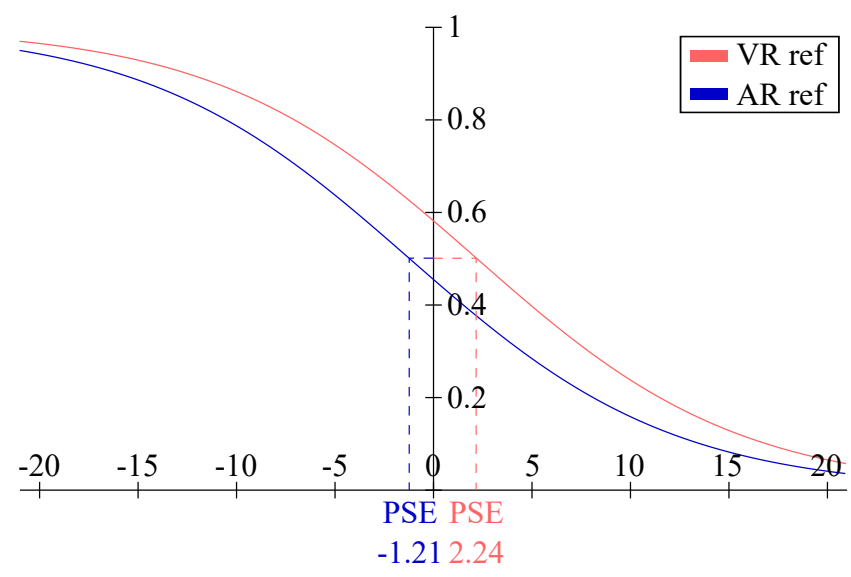

Figure 6: Psychometric curves for each condition. The red (resp. blue) curve shows the psychometric curve with VR (resp. AR) as a stiffness reference. The corresponding Point of Subjective Equality (PSE) is displayed for each condition. Curves were plotted using the dedicated psignifit software.

Due to the significance of $\mathbf{C 1}$, we further analyzed the recognition accuracy by fitting psychometric curves (see Equation 1 ) to the data based on the question: is the comparison object stiffer? We computed the curve for each level of $\mathbf{C 1}$ using the dedicated psignifit softwar ${ }^{3}$ (see Figure 6).

$$
f(x)=\frac{1}{1+\mathrm{e}^{-\frac{x-\alpha}{\beta}}}
$$

The obtained coefficients were $\alpha=2.24(C I=[0.03,4.06])$ and $\beta=-6.69(C I=[-8.09,-5.18])$ for the VR reference condition and $\alpha=-1.21(C I=[-3.18,0.6])$ and $\beta=-6.72$ $(C I=[-8.85,-5.84])$ for the AR reference condition. Such $\alpha$ coefficient determines a Point of Subjective Equality (PSE) which represents the value in which both pistons are considered to be equivalent (e.g. $50 \%$ of chance to choose one or another). The lower value of the PSE for the VR reference condition supports the ANOVA results on the significance of $\mathbf{C 1}$. The corresponding JND values were $11.09(C I=[7.89,14.44])$ for the VR condition and $11.14(C I=[9.09,14.9])$ for the AR condition.

\subsection{Response Time}

We also evaluated the influence of $\mathbf{C 1}$ and $\mathbf{C 2}$ on the time participants needed to answer $(\mathbf{O m} 2)$. The two-way ANOVA $\mathbf{C 1}$ and $\mathbf{C 2}$ vs. answering time did not show any significant effect. On average, participants needed $M=1.75 s ; S D=1.15 s$ to respond.

\subsection{Spring Displacement}

Regarding the total displacement applied (Om3), the two-way ANOVA C1 and C2 vs. displacement, showed a main effect on $\mathbf{C 1}\left(F_{1,11}=6.60 ; p<0.05 ; \eta_{p}^{2}=0.37\right)$. Yet, the relevance of this significance is limited due to the mean differences and the data variability: VR condition $M=0.217 \mathrm{~cm} ; S D=0.12 \mathrm{~cm}$, AR condition $M=0.225 \mathrm{~cm} ; S D=0.13 \mathrm{~cm}$. No main effect on $\mathbf{C 2}$ $\left(F_{4,44}=1.44 ; p=0.236 ; \eta_{p}^{2}=0.11\right)$ nor interaction effects were found $\left(F_{4,44}=2.00 ; p=0.111 ; \eta_{p}^{2}=0.15\right)$.

\subsection{Force Exertion}

Regarding the exerted force $(\mathbf{O m 4})$ (see Figure 7), the two-way ANOVA C1 and C2 vs. force, showed a main effect on C1 $\left(F_{1,11}=53.52 ; p<0.001 ; \eta_{p}^{2}=0.83\right)$ and on C2 $\left(F_{4,44}=35.82\right.$;

${ }^{3}$ https://github.com/wichmann-lab/psignifit/blob/master/psignifit.m

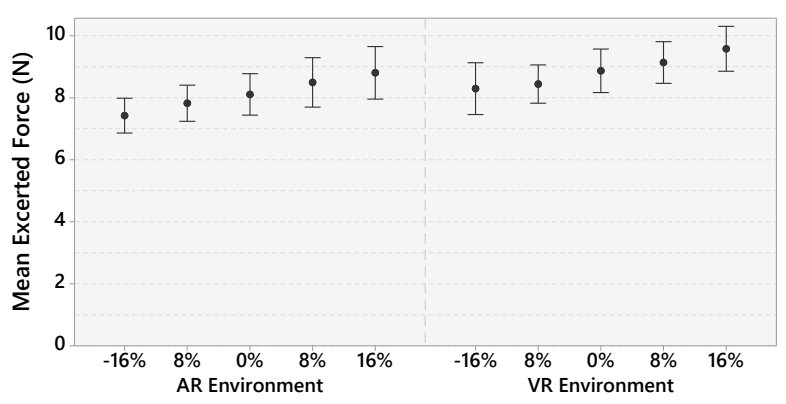

Figure 7: Mean exerted force for the five stiffness conditions and the two environment conditions.

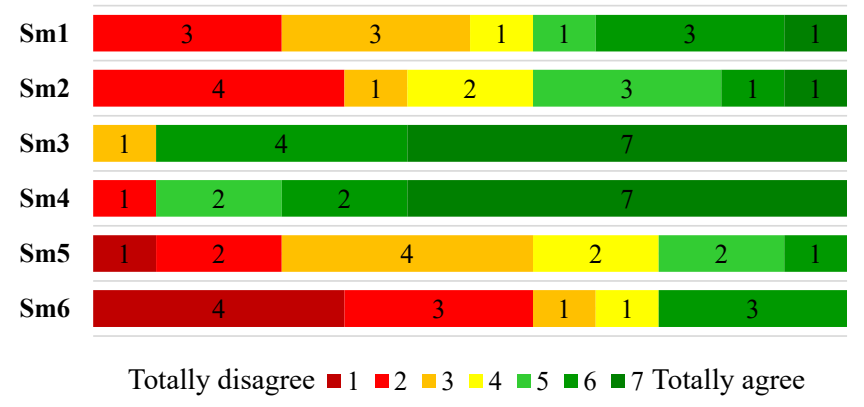

Figure 8: Subjective questionnaire results. Each line corresponds to the answer of the participants for a subjective measure, evaluated on a 7-point Likert scale. Green colors correspond to positive answers. Red colors correspond to negative answers.

$\left.p<0.001 ; \eta_{p}^{2}=0.77\right)$. Post-hoc tests showed that participants significantly exerted more force in the VR condition $(M=8.86 N$; $S D=1.18 N)$ than in the AR condition $(M=8.13 N ; S D=1.17 N)$ and that the exerted force increased with the stiffness of the spring. No interaction effects were found $\left(F_{4,44}=0.30 ; p=0.877 ; \eta_{p}^{2}=0.03\right)$.

\subsection{Subjective answers}

Figure 8 presents the answers collected through our subjective questionnaire (7-point Likert scale). Regarding the appearance of the virtual piston, participants reported that the virtual piston barely seemed real in AR $(\mathbf{S m 1}, M=4.08 ; S D=1.83)$ and in VR $(\mathbf{S m 2}$, $M=3.92 ; S D=1.73)$. A Student test showed this difference was not significant $(\mathrm{p}=0.55$, Qobs $=0.62)$.

Regarding the quality of the AR display, 11 participants reported that the virtual scene correctly occluded the real environment, 7 of which gave the maximal rank (Sm3, $M=6.33$; $S D=1.15)$. Participants did not perceive any strong difference between the real and the virtual scenes, 7 of them giving the maximal rank $(\mathbf{S m 4}, M=6.08$; $S D=1.51$ ). Two participants reported difference in luminosity between the two scenes, in favor of the virtual scene.

Five participants reported a positive or neutral visual fatigue during the experiment $(\mathbf{S m 5}, M=3.42 ; S D=1.44)$. One participant reported that the lighting in VR was tiring. Participants reported overall medium levels of fatigue (Sm6, $M=2.92 ; S D=2.07$ ), and 3 of them reported higher levels of fatigue. Concerning the last open question, 8 participants reported they did not think the environment influenced their haptic perception (Sm7). Two participants reported the piston felt softer in VR. One participant reported the piston felt softer in AR. 
To appear in an IEEE VGTC sponsored conference proceedings

\section{Discussion}

The results of our psychophysical study show a difference between the stiffness perceived in augmented reality and the stiffness perceived in virtual reality. The virtual piston was significantly more often perceived as stiffer in the VR condition than in the AR condition. In particular, given an equal stiffness between the two pistons in $\mathrm{AR}$ and VR, the participants on average reported that the piston was stiffer in the VR condition around $60 \%$ of the time. Moreover, the two computed Points of Subjective Equality (PSE) (between a reference piston tested in one condition and a comparison piston tested in the other condition) are different, suggesting a perceptual offset of $3.45 \%$ on average. Thus, taken together, our results suggest a psychological effect or bias, as if the piston tested in a purely virtual environment feels "stiffer", and the same piston tested in an augmented (real) environment feels "softer".

The JND values found in our experiment are around $11 \%$. This value is smaller than the one usually found in the literature for stiffness discrimination (between $15 \%$ and $22 \%$ ), and closer to the JND found for force discrimination (10\%). However, contrary to the participants in these studies, participants in our study could see the object, which makes the discrimination task easier, as already observed in [15].

From the subjective questionnaires, one can notice that the quality of our VR scene seems to be well appreciated, and estimated as a convincing reproduction of the real scene. The participants have indeed found that the real scene was well occluded by the virtual scene in the VR condition ( $\mathbf{S m 3}$ ). They also found very little difference between the AR and the VR scenes (Sm4). The participants reported low levels of visual (Sm5) or haptic (Sm6) fatigue after the experiment. We performed an additional analysis comparing recognition rates and answering times between the first and last blocks of the experiment. A Wilcoxon signed-rank test showed no difference, suggesting that - even though little - the reported fatigue did not influence the collected measures.

Surprisingly, almost all participants reported that the type of display (AR versus VR) did not influence their haptic perception (Sm7). This suggests that the participants were not aware at all that the visual condition had an influence on their answer. However, participant 3 reported that "The piston felt stiffer in VR because all elements are congruent, i.e. they are all virtuals".

Another interesting observation relates to the measures of forces and displacement applied on the virtual piston in the two conditions. There was no difference found in terms of quantity of displacement applied on the piston between the VR and AR conditions (Om3). However, the participants received $11 \%$ more force in the VR condition compare to AR (Om4). This means that the participants applied the same quantity of movement and probably kept on constantly applying oscillating pressures up and down. But they stopped their motion earlier in the AR condition and went "deeper" in the piston in the VR condition. As a result, they exerted and received more force when the scene was entirely virtual. This change in the exploration strategy could thus also explain the fact that the virtual piston in VR was perceived as stiffer. Another interpretation could here be that the participants felt "safer" in the virtual condition and/or "less confident" in the AR condition. In any case, this surprising difference in haptic interaction strategy - the fact that there is a greater motor involvement (and higher forces exertion) in the VR environment - calls for further behavioural studies. Future work is now necessary to deeper qualify how and why people have different exploration strategies, different ways of interacting, and different final haptic perception in such virtual versus augmented versus real environments.

Another aspect to consider is the realism of the scene. In our setup, there were some slight differences in appearance (colors, inter-object reflections) between the AR and VR scenes. While some of these differences stem from current technological limitations, a thorough investigation of the impact of the level of photo-realism on stiffness perception should also be investigated in future work.

\section{Conclusion}

We studied haptic perception in augmented reality versus in virtual reality. We designed an experimental setup based on a Microsoft HoloLens visual display and a force-feedback device. Participants could press on a virtual piston in either in an AR or in a VR environment, and compare their stiffness.

The results of our psychophysical study show that the participants have perceived the virtual piston as "stiffer" in the virtual environment than in the augmented environment. In the case of equivalent stiffness between AR and VR, participants chose the VR piston as the stiffer one in $60 \%$ of cases. We also found that the forces exerted by the participants on the virtual piston were higher in virtual reality than in augmented reality, suggesting different exploration strategies.

Taken together our results suggest that haptic perception of virtual objects is different in augmented reality compare to virtual reality. In particular, they suggest a new psychological phenomenon: a bias in haptic perception making virtual objects feel "softer" in augmented environments compare to purely virtual environments. These results could pave the way to future studies aiming at characterizing differences in perception between reality, augmented reality, and virtual reality.

\section{REFERENCES}

[1] S. A. Cholewiak, H. Z. Tan, and D. S. Ebert. Haptic identification of stiffness and force magnitude. In Proceedings of the IEEE Symposium on Haptic Interfaces for Virtual Environment and Teleoperator Systems, pages 87-91, 2008.

[2] M. Congedo, A. Lécuyer, and E. Gentaz. The influence of spatial delocation on perceptual integration of vision and touch. Presence: Teleoperators and Virtual Environments, 15(3):353-357, 2006.

[3] D. Drascic and P. Milgram. Perceptual issues in augmented reality. In Proceedings of Electronic Imaging: Science \& Technology, pages 123-134. International Society for Optics and Photonics, 1996.

[4] N. Gurari, K. J. Kuchenbecker, and A. M. Okamura. Stiffness discrimination with visual and proprioceptive cues. In Proceedings of the IEEE World Haptics Conference, pages 121-126, 2009.

[5] S. Hashiguchi, Y. Sano, F. Shibata, and A. Kimura. Rv dynamics illusion: Psychophysical influence on sense of weight by mixed-reality visual stimulation of moving objects. In Proceedings of the International Conference on Virtual, Augmented and Mixed Reality, pages 55-64. Springer, 2014.

[6] Y. Hirano, A. Kimura, F. Shibata, and H. Tamura. Psychophysical influence of mixed-reality visual stimulation on sense of hardness. In Proceedings of the IEEE International Conference on Virtual Reality, pages 51-54, 2011.

[7] S. Jeon and S. Choi. Modulating real object stiffness for haptic augmented reality. In Proceedings of the International Conference on Human Haptic Sensing and Touch Enabled Computer Applications, pages 609-618. Springer, 2008.

[8] J. A. Jones, J. E. Swan II, G. Singh, E. Kolstad, and S. R. Ellis. The effects of virtual reality, augmented reality, and motion parallax on egocentric depth perception. In Proceedings of the ACM Symposium on Applied Perception in Graphics and Visualization, pages 9-14, 2008.

[9] L. A. Jones and I. W. Hunter. A perceptual analysis of stiffness. Experimental Brain Research, 79(1):150-156, 1990.

[10] L. A. Jones and H. Z. Tan. Application of psychophysical techniques to haptic research. IEEE Transactions on Haptics, 6(3):268-284, 2013.

[11] B. Knorlein, M. Di Luca, and M. Harders. Influence of visual and haptic delays on stiffness perception in augmented reality. In Proceedings of the IEEE International Symposium on Mixed and Augmented Reality, pages 49-52, 2009.

[12] E. Kruijff, J. E. Swan, and S. Feiner. Perceptual issues in augmented reality revisited. In Proceedings of the IEEE International Symposium on Mixed and Augmented Reality, pages 3-12, 2010. 
[13] A. Lécuyer. Simulating haptic feedback using vision: A survey of research and applications of pseudo-haptic feedback. Presence: Teleoperators and Virtual Environments, 18(1):39-53, 2009.

[14] A. Lécuyer, J.-M. Burkhardt, S. Coquillart, and P. Coiffet. " boundary of illusion": an experiment of sensory integration with a pseudo-haptic system. In Proceedings of the IEEE International Conference on Virtual Reality, pages 115-122, 2001.

[15] A. Lécuyer, S. Coquillart, A. Kheddar, P. Richard, and P. Coiffet. Pseudo-haptic feedback: Can isometric input devices simulate force feedback? In Proceedings of the IEEE International Conference on Virtual Reality, pages 83-90, 2000.

[16] A. Pressman, L. J. Welty, A. Karniel, and F. A. Mussa-Ivaldi. Perception of delayed stiffness. The International Journal of Robotics Research, 26(11-12):1191-1203, 2007.

[17] P. Punpongsanon, D. Iwai, and K. Sato. Softar: Visually manipulating haptic softness perception in spatial augmented reality. IEEE Transactions on Visualization and Computer Graphics, 21(11):1279-1288, 2015.

[18] J. P. Rolland, W. Gibson, and D. Ariely. Towards quantifying depth and size perception in virtual environments. Presence: Teleoperators \& Virtual Environments, 4(1):24-49, 1995.

[19] M. A. Srinivasan, G. L. Beauregard, and D. L. Brock. The impact of visual information on the haptic perception of stiffness in virtual environments. In Proceedings of the ASME Dynamic Systems and Control Division, volume 58, pages 555-559, 1996.

[20] Y. Visell, K. A. Duraikkannan, and V. Hayward. A device and method for multimodal haptic rendering of volumetric stiffness. In Proceedings of the International Conference on Human Haptic Sensing and Touch Enabled Computer Applications, pages 478-486. Springer, 2014.

[21] W.-C. Wu, C. Basdogan, and M. A. Srinivasan. Visual, haptic, and bimodal perception of size and stiffness in virtual environments. In Proceedings of the ASME Dynamic Systems and Control Division, volume 67, pages 19-26, 1999.

[22] C. J. Ziemer, J. M. Plumert, J. F. Cremer, and J. K. Kearney. Estimating distance in real and virtual environments: Does order make a difference? Attention, Perception, \& Psychophysics, 71(5):1095-1106, 2009 